\title{
Temperature dependence of quantum correlations in 1D macromolecular chains
}

\author{
A. V. Chizhov ${ }^{1,2}$, D. Chevizovich ${ }^{3}$, Z. Ivić $^{3}$, S. Galović ${ }^{3}$ \\ ${ }^{1}$ Bogoliubov Laboratory of Theoretical Physics, Joint Institute for Nuclear Research, \\ Joliot-Curie, 6, Dubna, 141980, Russia \\ ${ }^{2}$ Dubna State University, Universitetskaya, 19, Dubna, 141980, Russia \\ ${ }^{3}$ Vinča Institute of Nuclear Sciences, 11001 Belgrade, Serbia \\ chizhov@theor.jinr.ru
}

PACS 05.60.Gg, 63.20.-e, 03.65.-w

DOI 10.17586/2220-8054-2019-10-2-141-146

We investigate the problem of generating quantum correlations between different sites of a macromolecular chain by vibronic excitation depending on the temperature. The influence of temperature on the model dynamics is taken into account by employing the partial-dressing method based on the modified LangFirsov unitary transformation under the assumption that the chain collective oscillations are in the thermal equilibrium state. To describe quantum correlations between the chain sites in the case of the initial single-vibronic excitation, we use two-time correlation functions of the second order and the logarithmic negativity as the degree of entanglement. We find that at certain temperatures for various model parameters time-stable entanglement can occur in the chain.

Keywords: energy transport, vibron, small polaron, correlation functions, entanglement.

Received: 2 February 2019

Revised: 28 February 2019

\section{Introduction}

The study of macromolecular chains is important from the point of view of the large role they play in biology and technology. In particular, it is believed that protein molecules act as mediators in the mechanism providing energy for diverse biological processes such as photochemical reactions, crossmembrane ion transfer and signal transduction, muscle contraction, cellular mobility [1]. Macromolecular systems, due to their inherent miniature characteristics, are considered as very promising materials for the manufacture of microelectronic and optoelectronic devices [2-4]. Recently, DNA molecules and various polymers have been used for recording and storing information which is important for the development of molecular and quantum computers [5-8].

The first explanation of the problem of energy transport along a polypeptide chain based on the quantum mechanics was proposed by Davydov [9,10]. Davydov's model supposed that, due to the exciton interaction with phonon modes, excitation forms a soliton that is more stable than the bare excitation. Nevertheless, due to the dipole-dipole coupling the life-time of the chain collective excitations appeared to be rather short. To overcome this problem, it was suggested that the energy losses may be prevented by the self-trapping mechanism caused by induced local distortion of the molecular chain. In this case, a vibronic excitation being surrounded by local lattice distortion may propagate in a polaron form along the chain with minimal energy losses for a long time [11,12].

One of the main tasks for implementing a quantum computer is the problem of creating sustainable entanglement. In this article, based on the concept of partial dressing, we analyze the possibility of generating quantum correlations in the molecular chain by vibronic excitation, as well as the conditions for their stable existence in time. We focus on studying the question of the temperatures at which long-lived entanglement can arise for different regimes of the molecular chain that can be helpful for using macromolecular systems in quantum computing.

\section{Model}

We consider a 1D macromolecular periodic polymer chain, in which the transfer of vibronic excitation from one molecule to neighboring molecules occurs due to hopping mechanism because of the resonance interaction between the molecules.

Then the Hamiltonian of the vibron and phonon subsystem has the form [10]:

$$
\begin{aligned}
\hat{H}= & \Delta \sum_{n} \hat{A}_{n}^{\dagger} \hat{A}_{n}-\sum_{n} J_{g} \hat{A}_{n}^{\dagger}\left(\hat{A}_{n+g}+\hat{A}_{n-g}\right)+\sum_{q} \hbar \omega_{q} \hat{B}_{q}^{\dagger} \hat{B}_{q} \\
& +\frac{1}{\sqrt{N}} \sum_{q} \sum_{n} F_{q} \mathrm{e}^{i q n R_{0}} \hat{A}_{n}^{\dagger} \hat{A}_{n}\left(\hat{B}_{q}+\hat{B}_{-q}^{\dagger}\right) .
\end{aligned}
$$


$\hat{A}_{n}$ is the vibron annihilation operator on the $n$-th lattice site, $\hat{B}_{q}$ is the phonon annihilation operator with the frequency $\omega_{q}, \Delta$ is the vibron excitation energy, $J_{g}$ are hopping constants, $R_{0}$ is a distance between sites in the chain, and $F_{q}$ is the vibron-phonon coupling parameter.

The transition to the polaron picture is achieved by applying the following unitary transformation operator (modified Lang-Firsov transformation) [13-15]:

$$
\hat{U}=\exp \left\{-\frac{1}{\sqrt{N}} \sum_{q} \sum_{n} f_{q} \mathrm{e}^{-i q n R_{0}} \hat{A}_{n}^{\dagger} \hat{A}_{n}\left(\hat{B}_{-q}-\hat{B}_{q}^{\dagger}\right)\right\} .
$$

Here $f_{q}=\delta \cdot F_{q}^{*} /\left(\hbar \omega_{q}\right)$, where $0 \leq \delta \leq 1$ is variational parameter which measures the degree of the vibron dressing.

In terms of such an operator one can introduce operators of new quasiparticles: dressed vibrons $\hat{a}_{n}=\hat{U} \hat{A}_{n} \hat{U}^{\dagger}$ $\left(\hat{a}_{n}^{\dagger}=\hat{U} \hat{A}_{n}^{\dagger} \hat{U}^{\dagger}\right)$, and new phonons $\hat{b}_{q}=\hat{U} \hat{B}_{q} \hat{U}^{\dagger}\left(\hat{b}_{q}^{\dagger}=\hat{U} \hat{B}_{q}^{\dagger} \hat{U}^{\dagger}\right)$.

Then the transformed Hamiltonian takes the form:

$$
\begin{aligned}
\hat{\tilde{H}} & =\hat{U} \hat{H} \hat{U}^{\dagger} \\
& =E \sum_{n} \hat{a}_{n}^{\dagger} \hat{a}_{n}-\sum_{n} J_{g} \hat{a}_{n}^{\dagger}\left(\hat{a}_{n+g} \hat{\Phi}_{n}(g)+\hat{a}_{n-g} \hat{\Phi}_{n}(-g)\right)+\sum_{q} \hbar \omega_{q} \hat{b}_{q}^{\dagger} \hat{b}_{q} \\
& +\frac{1}{\sqrt{N}} \sum_{q} \sum_{n}\left(F_{q}-\hbar \omega_{q} f_{q}^{*}\right) \mathrm{e}^{i q n R_{0}} \hat{a}_{n}^{\dagger} \hat{a}_{n}\left(\hat{b}_{q}+\hat{b}_{-q}^{\dagger}\right) \\
& +\frac{1}{N} \sum_{q}\left[\hbar \omega_{q}\left|f_{q}\right|^{2}-F_{q}\left(f_{q}+f_{-q}^{*}\right)\right] \sum_{n \neq n^{\prime}} \mathrm{e}^{i q R_{0}\left(n-n^{\prime}\right)} \hat{a}_{n}^{\dagger} \hat{a}_{n} \hat{a}_{n^{\prime}}^{\dagger} \hat{a}_{n^{\prime}},
\end{aligned}
$$

where

$$
\begin{aligned}
E & =\Delta-\frac{1}{N} \sum_{q}\left[F_{q}\left(f_{q}+f_{-q}^{*}\right)-\hbar \omega_{q}\left|f_{q}\right|^{2}\right], \\
\hat{\Phi}_{n}(g) & =\exp \left\{\frac{1}{\sqrt{N}} \sum_{q} f_{q} \mathrm{e}^{-i q n R_{0}}\left(\hat{b}_{-q}-\hat{b}_{q}^{\dagger}\right)\left(\mathrm{e}^{-i q R_{0} g}-1\right)\right\} .
\end{aligned}
$$

In order to account for the influence of the thermal fluctuations on the properties of the autolocalized vibron, we apply the mean-field approach by averaging the transformed Hamiltonian over the phonon subsystem. Defining exciton states in the representation of wave vectors $k$, which take $N$ discrete values, $k=2 \pi \nu(\nu=0, \pm 1, \pm 2 \ldots)$ in the interval $-\pi / R_{0}<k \leq \pi / R_{0}$, by:

$$
\hat{a}_{k}=(1 / \sqrt{N}) \sum_{n} \mathrm{e}^{-i k n R_{0}} \hat{a}_{n}
$$

an effective mean-field Hamiltonian reads:

$$
\hat{H}_{\mathrm{MF}}=\sum_{k} E_{\mathrm{SP}}(k) \hat{a}_{k}^{\dagger} \hat{a}_{k}+\sum_{q} \hbar \omega_{q} \hat{b}_{q}^{\dagger} \hat{b}_{q}
$$

with the energy of the small-polaron band state:

$$
E_{\mathrm{SP}}(k)=\Delta-\frac{1}{N} \sum_{q}\left[F_{q}\left(f_{q}+f_{-q}^{*}\right)-\hbar \omega_{q}\left|f_{q}\right|^{2}\right]-2 J_{g} \mathrm{e}^{-W_{g}(T)} \cos \left(g k R_{0}\right),
$$

where:

$$
W_{g}(T)=\frac{1}{N} \sum_{q}\left|f_{q}\right|^{2}\left(2\left\langle n_{q}(T)\right\rangle+1\right)\left(1-\cos \left(g q R_{0}\right)\right)
$$

is the vibron-band narrowing factor (which characterizes the enhancement of the polaron effective mass), and the phonon average number $\left\langle n_{q}(T)\right\rangle=1 /\left(\mathrm{e}^{\hbar \omega_{q} / k_{\mathrm{B}} T}-1\right)$.

Using Hamiltonian (7) and (inverse) transformation (6) we can determine the time evolution of the vibronic operators:

$$
\hat{a}_{n}(t)=(1 / \sqrt{N}) \sum_{k} \mathrm{e}^{i\left[k n R_{0}-\omega_{k} t\right]} \hat{a}_{k}(0)=\sum_{m} \hat{a}_{m}(0) \cdot(1 / N) \sum_{k} \mathrm{e}^{i\left[k(n-m) R_{0}-\omega_{k} t\right]},
$$

where $\omega_{k}=E_{\mathrm{SP}}(k) / \hbar$.

In order to fix the variational parameter $\delta$ at temperature $T$ with certain model parameters, we use the procedure of minimization of $E_{\mathrm{SP}}$. In terms of adiabatic parameter $B=2|J| /\left(\hbar \omega_{C}\right)$ and coupling constant $S=E_{\mathrm{B}} /\left(\hbar \omega_{C}\right)$ 
(where $E_{\mathrm{B}}=(1 / N) \sum_{q}\left\{\left|F_{q}\right|^{2} /\left(\hbar \omega_{q}\right)\right\}$ is the lattice deformation energy and Debye frequency $\omega_{C}$ ) the problem of minimization of $E_{\mathrm{SP}}$ reduces effectively to minimization of the function:

$$
\mathcal{E}(\tau)=-S(2-\delta) \delta-B \mathrm{e}^{-\delta^{2} W_{g}(\tau, S)},
$$

where $\tau=k_{\mathrm{B}} T /\left(\hbar \omega_{C}\right)$ is the normalized temperature. Thus, the variational parameter $\delta$ at temperature $T$ is found by the conditions $\partial \mathcal{E}(\tau) / \partial \delta=0$ and $\partial^{2} \mathcal{E}(\tau) / \partial \delta^{2}>0$. This minimization procedure is similar to that used in refs. [16-18] for the study of polaron dressing in various systems of molecular chains.

The behavior of parameter $\delta$ for three different temperatures is shown in Fig. 1. It is important to note here that, along with the areas of smooth variation of the parameter, there is also an area of its sharp change. Since the variational parameter measures the degree of vibron dressing, such a sudden change of the value of $\delta$ may indicate an abrupt change of the degree of vibron dressing, i.e., a sudden change of its dynamical nature, which can be related to the phenomenon of the polaron crossover.
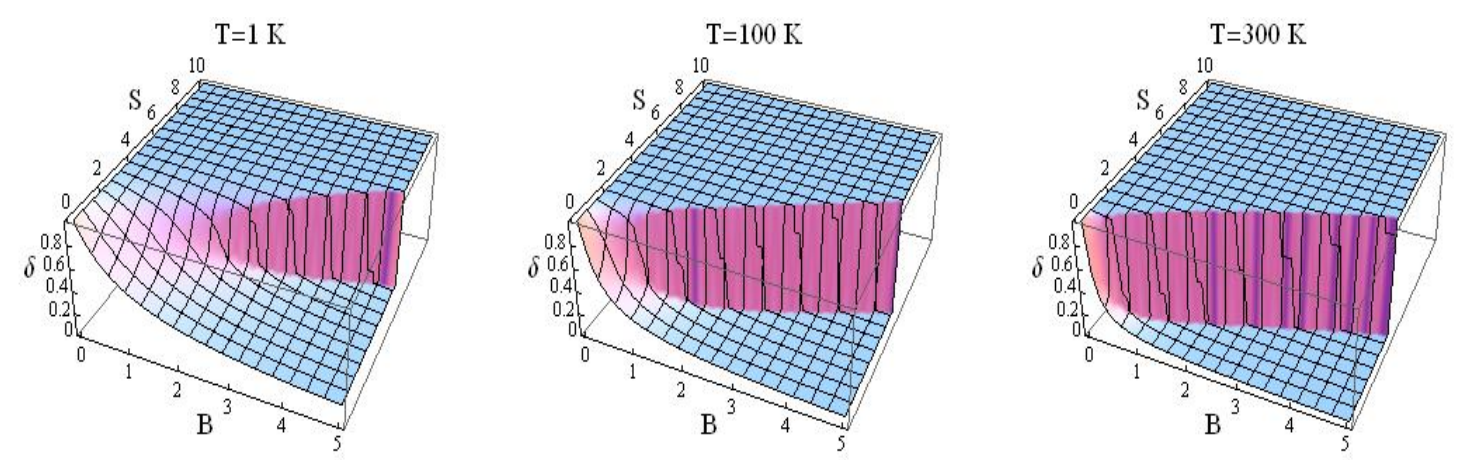

FIG. 1. Vibron dressing factor for $T=1 \mathrm{~K}, T=100 \mathrm{~K}$, and $T=300 \mathrm{~K}\left(\omega_{C}=10^{13} \mathrm{~s}^{-1}\right)$

The temperature dependence of the energy of a small polaron obtained in this way allows us to investigate quantum correlations that can occur between various sites of the molecular chain after the excitation of one of these sites.

\section{Correlation functions}

One of the methods for studying quantum correlations is to use correlation function [19]. In the case of single-vibronic excitation of the molecular chain, considered in this paper, full information on quantum correlations will be determined by the two-time correlation function of the second order which is defined as:

$$
G^{(1,1)}\left(m, t_{1} ; n, t_{2}\right)=\operatorname{Tr}\left\{\varrho \hat{a} \hat{a}_{m}^{\dagger}\left(t_{1}\right) \hat{a}_{n}\left(t_{2}\right)\right\}
$$

where $\hat{\varrho}$ is the density matrix of the initial intramolecular excitation of the chain. This function determines the degree of the quantum (cross)correlation between the $m$-th and $n$-th sites of the chain, respectively, at times $t_{1}$ and $t_{2}$. In addition, the function $G^{(1,1)}\left(m, t_{1} ; m, t_{2}\right)$ corresponds to the auto-correlation of the $m$-th site at times $t_{1}$ and $t_{2}$.

The initial density matrix of the chain in the case of a single-vibronic excitation can be determined in the basis of eigenstates of number operators corresponding to the chain sites $\hat{N}_{n}(0)=\hat{A}_{n}^{\dagger}(0) \hat{A}_{n}(0)=\hat{a}_{n}^{\dagger}(0) \hat{a}_{n}(0)=\hat{n}_{n}(0)$. If at the initial moment a single-vibration excitation has arisen, e.g., at the $l$-th site of the chain, then the density matrix can be represented in the form:

$$
\hat{\varrho}=\left|1_{l}\right\rangle\left\langle 1_{l}\left|=\hat{a}_{l}^{\dagger}(0)\right|\{0\}\right\rangle\langle\{0\}| \hat{a}_{l}(0),
$$

where $|\{0\}\rangle$ is the chain vacuum state when all sites are in unexcited (ground) states.

Then, taking into account Eqs. (10) and (13), the correlation function (12) is brought to the form:

$$
\begin{aligned}
& G^{(1,1)}\left(m, t_{1} ; n, t_{2} \mid 1_{l}\right)=\left\langle 1_{l}\left|\hat{a}_{m}^{\dagger}\left(t_{1}\right) \hat{a}_{n}\left(t_{2}\right)\right| 1_{l}\right\rangle \\
& =\frac{1}{N} \sum_{k} \exp \left\{-i\left[k R_{0}(m-l)-\omega_{k} t_{1}\right]\right\} \cdot \frac{1}{N} \sum_{k^{\prime}} \exp \left\{i\left[k^{\prime} R_{0}(n-l)-\omega_{k^{\prime}} t_{2}\right]\right\} .
\end{aligned}
$$


In macromolecules it is assumed that $N \gg 1$, so in the limit $N \rightarrow \infty$ one can use the following limiting relation

It means that:

$$
\frac{1}{N} \sum_{k} \ldots \rightarrow \frac{R_{0}}{2 \pi} \int_{-\pi / R_{0}}^{\pi / R_{0}} d k \ldots
$$

$$
\begin{aligned}
& \frac{1}{N} \sum_{k} \mathrm{e}^{i\left[k R_{0}(m-n)-\omega_{k} t\right]} \rightarrow \frac{R_{0}}{2 \pi} \int_{-\pi / R_{0}}^{\pi / R_{0}} d k \exp \left\{i\left[k R_{0}(m-n)-\omega_{k} t\right]\right\} \\
& =i^{m-n} \mathrm{e}^{-i\left[\Delta / \hbar-\omega_{C} S(2-\delta) \delta\right] \cdot t} J_{m-n}\left(t \omega_{C} B \mathrm{e}^{-\delta^{2} S \operatorname{coth}(1 / 2 \tau)}\right) \equiv c_{m n}(t),
\end{aligned}
$$

where $J_{n}(x)$ is the Bessel function of the first kind of order $n$. Thus, the correlation function (14) can be given as:

$$
G^{(1,1)}\left(m, t_{1} ; n, t_{2} \mid 1_{l}\right)=c_{m l}^{*}\left(t_{1}\right) c_{n l}\left(t_{2}\right)
$$

\section{Entanglement}

As the measure of the degree of entanglement of bipartite states one can use the logarithmic negativity [20]:

$$
E_{\mathcal{N}}(\hat{\varrho})=\log _{2}\left\|\hat{\varrho}^{T_{2}}\right\|
$$

based on the trace norm:

$$
\left\|\varrho^{T_{2}}\right\|=\operatorname{Tr} \sqrt{\left(\varrho^{T_{2}}\right)^{\dagger} \hat{\varrho}^{T_{2}}}
$$

of the partial transpose of the density matrix of a bipartite state:

$$
\left\langle i, j\left|\varrho^{T_{2}}\right| k, l\right\rangle=\langle i, l|\varrho| k, j\rangle .
$$

For entangled states:

$$
|| \hat{\varrho}_{\text {ent }}^{T_{2}} \|=1+2\left|\sum_{i} \mu_{i}\right| \equiv 1+2 \mathcal{N}(\hat{\varrho})>1
$$

where $\mu_{i}<0$ are negative eigenvalues of $\hat{\varrho}_{\text {ent }}^{T_{2}}$ so that

$$
E_{\mathcal{N}}\left(\hat{\varrho}_{\text {ent }}\right)>0 \text {. }
$$

The partial transpose of the reduced density matrix for the sites $m$ and $n$ in the representation of the state vectors $(|10\rangle,|01\rangle,|00\rangle,|11\rangle)$ can be represented in the matrix form:

$$
\hat{\varrho}_{\{m n\}}^{T_{n}}(t)=\left(\begin{array}{cccc}
\left|c_{l m}(t)\right|^{2} & 0 & 0 & 0 \\
0 & \left|c_{l n}(t)\right|^{2} & 0 & 0 \\
0 & 0 & 1-\left|c_{l m}(t)\right|^{2}-\left|c_{l n}(t)\right|^{2} & c_{l n}^{*}(t) c_{l m}(t) \\
0 & 0 & c_{l m}^{*}(t) c_{l n}(t) & 0
\end{array}\right)
$$

which has only one negative eigenvalue:

$$
\lambda_{N}=\frac{1}{2}\left[1-\left|c_{l m}(t)\right|^{2}-\left|c_{l n}(t)\right|^{2}-\sqrt{\left(1-\left|c_{l m}(t)\right|^{2}-\left|c_{l n}(t)\right|^{2}\right)^{2}+4\left|c_{l m}(t)\right|^{2}\left|c_{l n}(t)\right|^{2}}\right],
$$

so that the logarithmic negativity:

$$
\begin{aligned}
E_{\mathcal{N}} & =\log _{2}\left[1+2\left|\lambda_{N}\right|\right] \\
& =\log _{2}\left[\left|c_{l m}(t)\right|^{2}+\left|c_{l n}(t)\right|^{2}+\sqrt{\left(1-\left|c_{l m}(t)\right|^{2}-\left|c_{l n}(t)\right|^{2}\right)^{2}+4\left|c_{l m}(t)\right|^{2}\left|c_{l n}(t)\right|^{2}}\right] .
\end{aligned}
$$

\section{Results}

The behavior of two-site correlation functions and the logarithmic negativity as functions of time and temperature for various model parameters are shown in Figs. 2 and 3. These figures demonstrate the appearance of quantum correlations between chain sites in the processes of energy transfer along the macromolecular chain. Although both figures look similar, however, the scale of the logarithmic negativity amplitude corresponds to the measure of entanglement degree. It is worth noting that at certain temperatures two-site quantum correlations in the chain for various model parameters $S$ and $B$ can become long-lived in time. This is due to the fact that with such system parameters, the condition of full dressing of the vibron is ensured $(\delta \sim 1)$ which effectively suppresses the parameter $B$ in the time-dependent argument of the Bessel function in Eq. (16) due to the exponential multiplier. Such a temperature decreases with increasing the coupling constant $S$ and decreasing the adiabatic parameter $B$. Thus, in the strong-coupling non-adiabatic regime $(S \gg 1$ and $B \ll 1)$, time-stable quantum correlations in the chain can occur at low temperatures, while in the weak-coupling adiabatic regime, the entanglement state in 
the chain can be maintained at high temperatures. This fact can be used to create optimal conditions for stable entanglement in such macromolecular systems.
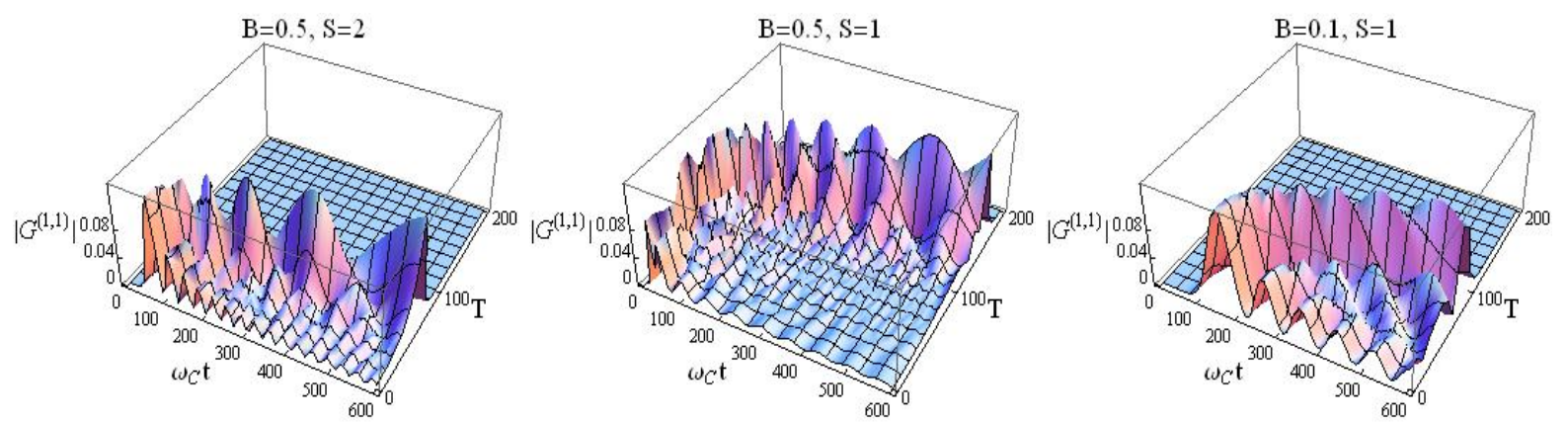

FIG. 2. Absolute value of the equal-time correlation function (17) for the sites $l-m=n-l=5$ depending on the scaled time $\left(\omega_{C}=10^{13} \mathrm{~s}^{-1}\right)$ and temperature (in Kelvin) at various model parameters
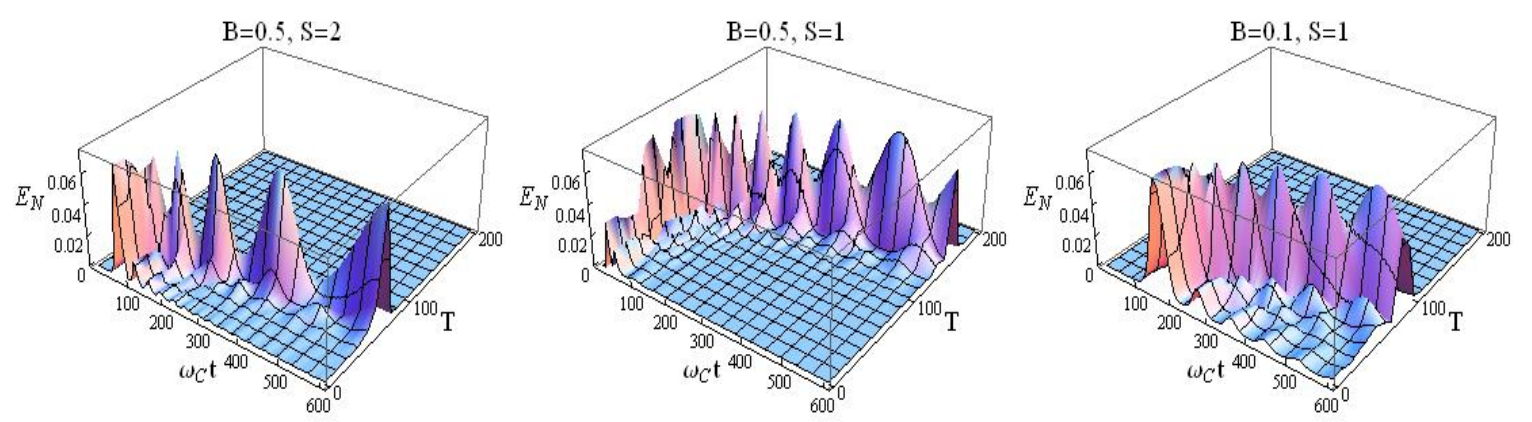

FIG. 3. The same as in Fig. 2 for the logarithmic negativity (25)

\section{Conclusion}

We analyzed the problem of generating quantum correlations between different chain sites depending on the temperature within the model of vibronic excitation transfer along a macromolecular chain. In order to take into account the effect of temperature on the model dynamics we used the partial-dressing method to treat the interaction problem of a vibron with chain collective oscillations being in the thermal equilibrium state. Based on two-time correlation functions of the second order and the logarithmic negativity as the degree of entanglement, in the case of the initial single-vibronic excitation we found occurrence of time-stable quantum correlations between the chain sites at certain temperatures for various model parameters. Such a temperature was shown to decrease with increasing the coupling constant and decreasing the adiabatic parameter that can be used to create required regimes for stable entanglement in macromolecular systems.

\section{Acknowledgements}

This work is partly supported by the bilateral project between the Serbian Ministry of Education and Science and "Theory of Condensed Matter" at JINR, Dubna, and by Serbian Ministry of Education and Science, under Contract Nos. III-45010 and III-45005.

\section{References}

[1] Davydov A. S. Biology and quantum mechanics. Naukova Dumka, Kiev, 1979, 142-216 (in Russian).

[2] Dekker C., Ratner M. A. Electronic properties of DNA. Phys. World, 2001, 14(8), P. 29-33.

[3] Conwell E. Polarons and transport in DNA. Topics in Current Chemistry, 2004, 237, P. 73-102.

[4] Mirkin C. A., Letsinger R. L., Mucic R. C., Storhoff J. J. A DNA-based method for rationally assembling nanoparticles into macroscopic materials. Nature, 1996, 382(6592), P. 607-609. 
[5] Adleman L. M. Molecular computation of solutions to combinatorial problems. Science, 1994, 266(5187), P. 1021-1024.

[6] Kahan M., Gil B., Adar R., Shapiro E. Towards molecular computers that operate in a biological environment. Physica D: Nonlinear Phenomena, 2008, 237(9), P. 1165-1172.

[7] Goldman N., Bertone P., Chen S., Dessimoz C., Leproust E. M., Sipos B., Birney E. Towards practical, high-capacity, low-maintenance information storage in synthesized DNA. Nature, 2013, 494(7435), P. 77-80.

[8] Kumar S. N. A proper approach on DNA based computer. American Journal of Nanomaterials, 2015, 3(1), P. 1-14.

[9] Davydov A. S. The theory of contraction of proteins under their excitation. J. Theor. Biol, 1973, 38(3), P. 559-569.

[10] Davydov A. S. Solitons in molecular systems. Phys. Scr., 1979, 20(2), P. 387-394.

[11] Lang I. G., Firsov Yu. A. Kinetic theory of semiconductors with low mobility. ZhETF, 1962, 43(5/11), P. 1843-1860.

[12] Yarkony D., Silbey R. Comments on exciton phonon coupling: Temperature dependence. J. Chem. Phys., 1976, 65(3), P. 1042-1052.

[13] Brown D. W., Ivić Z. Unification of polaron and soliton theories of exciton transport. Phys. Rev. B, 1989, 40(14), P. 9876-9887.

[14] Čevizović D., Galović S., Ivić Z. Nature of the vibron self-trapped states in hydrogenbonded macromolecular chains. Phys. Rev. E, 2011, 84(1), P. 011920-6.

[15] Čevizović D., Galović S., Reshetnyak A., Ivić Z. Vibron self-trapped states in biological macromolecules: Comparison of different theoretical approaches. J. Phys.: Conf. Ser, 2012, 393(1), P. 012033-8.

[16] Čevizović D., Galović S., Reshetnyak A., Ivić Z. The vibron dressing in $\alpha$-helicoidal macromolecular chains. Chin. Phys. B, 2013, 22(6), P. 060501-10

[17] Čevizović D., Ivić Z, Galović S., Reshetnyak A., Chizhov A. On the vibron nature in the system of two parallel macromolecular chains: The influence of interchain coupling. Physica B, 2016, 490, P. 9-15.

[18] Čevizović D., Chizhov A. V., Galović S. Vibron transport in macromolecular chains with squeezed phonons. Nanosystems: Physics, Chemistry, Mathematics, 2018, 9(5), P. 597-602.

[19] Glauber R. J. The quantum theory of optical coherence. Phys. Rev., 1963, 130(6), P. 2529-2539.

[20] Vidal G., Werner R. F. Computable measure of entanglement. Phys. Rev. A, 2002, 65(3), P. 032314-11. 